\title{
ORBITAL DIGRAPHS OF INFINITE PRIMITIVE PERMUTATION GROUPS
}

\author{
SIMON M. SMITH
}

\begin{abstract}
If $G$ is a group acting on a set $\Omega$ and $\alpha, \beta \in \Omega$, the digraph whose vertex set is $\Omega$ and whose arc set is the orbit $(\alpha, \beta)^{G}$ is called an orbital digraph of $G$. Each orbit of the stabiliser $G_{\alpha}$ acting on $\Omega$ is called a suborbit of $G$.

A digraph is locally finite if each vertex is adjacent to at most finitely many other vertices. A locally finite digraph $\Gamma$ has more than one end if there exists a finite set of vertices $X$ such that the induced digraph $\Gamma \backslash X$ contains at least two infinite connected components; if there exists such a set containing precisely one element, then $\Gamma$ has connectivity one.

In this paper we show that if $G$ is a primitive permutation group whose suborbits are all finite, possessing an orbital digraph with more than one end, then $G$ has a primitive connectivity-one orbital digraph, and this digraph is essentially unique. Such digraphs resemble trees in many respects, and have been fully characterised in a previous paper by the author.
\end{abstract}

This is a post-print repository version of the paper: Orbital digraphs of infinite primitive permutation groups, J. Group Theory 10 (2007). The final publication is available at www.referenceglobal.com by following this link: https://doi.org/10.1515/JGT. 2007.060

\section{Preliminaries}

Throughout this paper, $G$ will be a group of permutations of an infinite set $\Omega$. A transitive group $G$ is primitive on $\Omega$ if the only $G$-invariant equivalence relations admitted by $\Omega$ are the trivial and universal relations. A transitive group $G$ is said to act regularly on $\Omega$ if $G_{\alpha}=1$ for each $\alpha \in \Omega$.

If $\alpha \in \Omega$ and $g \in G$, we denote the image of $\alpha$ under $g$ by $\alpha^{g}$. Following this notation, all permutations will act on the right. The set of images of $\alpha$ under all elements of $G$ is called an orbit of $G$, and is denoted by $\alpha^{G}$. There is a natural action of $G$ on the $n$ element subsets and $n$-tuples of $\Omega$ via $\left\{\alpha_{1}, \ldots, \alpha_{n}\right\}^{g}:=\left\{\alpha_{1}^{g}, \ldots, \alpha_{n}^{g}\right\}$ and $\left(\alpha_{1}, \ldots, \alpha_{n}\right)^{g}:=\left(\alpha_{1}^{g}, \ldots, \alpha_{n}^{g}\right)$ respectively.

Date: 2007.

1991 Mathematics Subject Classification. 20 B 15; 05 C 25.

Email: simon.smith@chch.oxon.org. 
If $\alpha \in \Omega$ we denote the stabiliser of $\alpha$ in $G$ by $G_{\alpha}$, and if $\Sigma \subseteq \Omega$ we denote the setwise and pointwise stabilisers of $\Sigma$ in $G$ by $G_{\{\Sigma\}}$ and $G_{(\Sigma)}$ respectively.

A digraph $\Gamma$ will be a directed graph without multiple edges or loops; it is a pair $(V \Gamma, A \Gamma)$, where $V \Gamma$ is the set of vertices and $A \Gamma$ the set of arcs of $\Gamma$. The set $A \Gamma$ consists of ordered pairs of distinct elements of $V \Gamma$. Two vertices $\alpha, \beta \in V \Gamma$ are adjacent if either $(\alpha, \beta)$ or $(\beta, \alpha)$ lies in $A \Gamma$. A digraph is locally finite if every vertex is adjacent to at most finitely many vertices. All paths will be undirected, unless otherwise stated. A digraph is infinite if its vertex set is infinite. The distance between two connected vertices $\alpha$ and $\beta$ in $\Gamma$ is denoted by $d_{\Gamma}(\alpha, \beta)$.

A graph is a pair $(V \Gamma, E \Gamma)$, in which $E \Gamma$ is a set of unordered pairs of elements of $V \Gamma$, each of which is known as an edge of $\Gamma$. The results presented in this paper concern digraphs, and are thus applicable to graphs.

The automorphism group of $\Gamma$ will be denoted by Aut $\Gamma$. A graph or digraph is primitive if Aut $\Gamma$ is primitive on the set $V \Gamma$, and is automorphism-regular if Aut $\Gamma$ acts regularly on $V \Gamma$.

If $G$ is a group of permutations of a set $\Omega$ and $\alpha, \beta \in \Omega$, then the digraph with vertex set $\Omega$ and $\operatorname{arcset}(\alpha, \beta)^{G}$ is called an orbital digraph of $G$. Note such digraphs are necessarily arc-transitive. The orbital digraph $\left(\Omega,(\alpha, \beta)^{G}\right)$ is non-diagonal if $\alpha$ and $\beta$ are distinct. Naturally, the graph $\left(\Omega,\{\alpha, \beta\}^{G}\right)$ is called an orbital graph of $G$.

The connected components of any digraph $\Gamma$ are Aut $\Gamma$-invariant equivalence classes on the vertex set of $\Gamma$; thus any non-diagonal primitive orbital digraph is connected.

\section{ENDS OF GRAPHS}

A concept that is fundamental to the arguments contained herein is that of an end. A half-line $L$ of a graph or digraph $\Gamma$ is a one-way infinite cycle-free path $x_{1} x_{2} x_{3} \ldots$ in $\Gamma$. The vertex $x_{1}$ is commonly known as the root of $L$. There is a natural equivalence relation on the set of half-lines of $\Gamma$ : two half-lines $L_{1}$ and $L_{2}$ are equivalent if there exist an infinite number of pairwise-disjoint paths connecting a vertex in $L_{1}$ to a vertex in $L_{2}$. The equivalence classes of this relation are called the ends of $\Gamma$. An end is called thick if it contains infinitely many disjoint half-lines, otherwise it is called thin. By convention, a finite graph or digraph has no ends.

Theorem 2.1. ([8, Corollary 1.5]) If $\Gamma$ is an infinite locally finite connected vertex-transitive graph then $\Gamma$ has 1,2 or $2^{\aleph_{0}}$ ends.

Given a digraph $\Gamma$, it is possible to construct an associated graph $\Gamma^{\prime}$, whose vertex set is $V \Gamma$, with two vertices being adjacent in $\Gamma^{\prime}$ if and only if they are adjacent in $\Gamma$. Clearly, the ends of a digraph and its 
associated graph are the same. Thus, the above theorem is also true of digraphs.

Much of what is currently known about graphs with more than one end is underpinned by the work of Dicks and Dunwoody in their seminal text [1]. An accessible summary of their results pertaining to locally finite graphs can be found in [3].

The connectivity of an infinite connected graph or digraph $\Gamma$ is the smallest possible cardinality of a subset $W$ of $V \Gamma$ for which the induced digraph $\Gamma \backslash W$ is not connected. A lobe of $\Gamma$ is a connected subgraph that is maximal subject to the condition it has connectivity strictly greater than one. If $\Gamma$ has connectivity one, then the vertices $\alpha$ for which $\Gamma \backslash\{\alpha\}$ is not connected are called the cut vertices of $\Gamma$.

Let $V_{1}$ be the set of cut vertices of $\Gamma$, and let $V_{2}$ be a set in bijective correspondence with the set of lobes of $\Gamma$. Define $T$ to be a bipartite digraph whose parts are $V_{1}$ and $V_{2}$. Two vertices $\alpha \in V_{1}$ and $x \in V_{2}$ are adjacent in $T$ if and only if $\alpha$ is contained in the lobe of $\Gamma$ corresponding to $x$; in this case there is a pair of arcs between them, one in each direction. This construction yields a tree, which is called the block-cutvertex tree of $\Gamma$. If $\Gamma$ has connectivity one and block-cut-vertex tree $T$, then for any group $G$ acting on $\Gamma$, there is a natural action on $T$.

Two digraphs $\Gamma_{1}$ and $\Gamma_{2}$ are quasi-isometric if there exists a map $\phi: V \Gamma_{1} \rightarrow V \Gamma_{2}$ and there exist constants $a, b>0$ such that for all $\alpha, \beta \in V \Gamma$ we have

$$
\frac{1}{a} d_{\Gamma_{1}}(\alpha, \beta)-b \leq d_{\Gamma_{2}}(\phi(\alpha), \phi(\beta)) \leq a . d_{\Gamma_{1}}(\alpha, \beta)+b .
$$

In [2, Proposition 1], Möller shows if two locally finite connected graphs are quasi-isometric, then there is a natural bijection between their ends. This is also true of digraphs.

Lemma 2.2. If $G$ is a primitive group of permutations of an infinite set $\Omega$ and $\Gamma_{1}$ and $\Gamma_{2}$ are two non-diagonal locally finite orbital digraphs of $G$ then there is a natural bijection between the ends of $\Gamma_{1}$ and the ends of $\Gamma_{2}$.

Proof. Suppose both $\Gamma_{1}=\left(\Omega,\left(\alpha_{1}, \beta_{1}\right)^{G}\right)$ and $\Gamma_{2}=\left(\Omega,\left(\alpha_{2}, \beta_{2}\right)^{G}\right)$ are locally finite orbital digraphs of $G$. Put $m_{1}:=d_{\Gamma_{1}}\left(\alpha_{2}, \beta_{2}\right)$ and $m_{2}:=$ $d_{\Gamma_{2}}\left(\alpha_{1}, \beta_{1}\right)$, and let $a:=\max \left\{m_{1}, m_{2}\right\}$. Since both $\Gamma_{1}$ and $\Gamma_{2}$ are connected, any arc in $\Gamma_{2}$ corresponds to a path of length $m_{1}$ in $\Gamma_{1}$, and any arc in $\Gamma_{1}$ corresponds to a path of length $m_{2}$ in $\Gamma_{2}$. Thus, if $\alpha, \beta \in \Omega$ then $d_{\Gamma_{1}}(\alpha, \beta) \leq m_{1} . d_{\Gamma_{2}}(\alpha, \beta) \leq a . d_{\Gamma_{2}}(\alpha, \beta)$, and $d_{\Gamma_{2}}(\alpha, \beta) \leq$ $m_{2} \cdot d_{\Gamma_{1}}(\alpha, \beta) \leq a \cdot d_{\Gamma_{1}}(\alpha, \beta)$. Therefore $(1 / a) d_{\Gamma_{1}}(\alpha, \beta) \leq d_{\Gamma_{2}}(\alpha, \beta) \leq$ a.d $d_{\Gamma_{1}}(\alpha, \beta)$, so $\Gamma_{1}$ and $\Gamma_{2}$ are quasi-isometric.

Henceforth, no distinction will be made between an end of $\Gamma_{1}$ and its corresponding end in $\Gamma_{2}$. Furthermore, we define a permutation-end of $G$ to be an end of an orbital digraph of $G$. By the above lemma, the set 
of permutation-ends of $G$ is equal to the set of ends of a non-diagonal orbital digraph of $G$, and is independent of the orbital digraph chosen.

\section{ThE CANONICAL ORBITAL DigRAPH}

In [4], Möller shows every connected locally finite primitive graph with more than one end is closely related to one with connectivity one.

Theorem 3.1. ([3, Theorem 15]) If $\Gamma$ is a connected locally finite primitive graph with more than one end then there exist vertices $\alpha, \beta \in V \Gamma$ such that the graph $\left(V \Gamma,\{\alpha, \beta\}^{\text {Aut } \Gamma}\right)$ has connectivity one and each lobe has at most one end.

Since any infinite vertex-transitive connectivity-one digraph has infinitely many ends, the following may be deduced.

Corollary 3.2. If $\Gamma$ is a locally finite primitive arc-transitive digraph then $\Gamma$ has 0,1 or $2^{\aleph_{0}}$ ends.

If $G$ is a group of permutations of a set $\Omega$, then the orbits of the action of the stabiliser $G_{\alpha}$ on $\Omega$ are called the $\alpha$-suborbits of $G$. The cardinality of a suborbit is called a subdegree of $G$.

Recall that the set of permutation-ends of a primitive group with no infinite subdegree is equal to the set of ends of any one of its nondiagonal orbital digraphs. A consequence of the above corollary therefore, is that infinite primitive groups with no infinite subdegree either have precisely one permutation-end, or they have uncountably many.

We can use Theorem 3.1 to tell us a great deal about the orbital digraphs of infinite primitive permutation groups.

Theorem 3.3. If $G$ is a primitive group of permutations of an infinite set $\Omega$, with more than one permutation-end and no infinite subdegree, then $G$ has a locally finite orbital digraph with connectivity one in which each lobe has at most one end.

The proof of this theorem relies on the following five lemmas. Let $G$ be a primitive group of permutations of an infinite set $\Omega$.

Lemma 3.4. If $G$ has a locally finite orbital digraph with more than one end then $G$ has an orbital digraph with connectivity one.

Proof. Suppose $G$ has a locally finite orbital digraph $\Gamma$ with more than one end. Let $\Gamma^{\prime}$ be the graph associated with $\Gamma$. By Theorem 3.1, we may find vertices $\alpha, \beta \in \Omega$ such that the graph $\left(\Omega,\{\alpha, \beta\}^{\text {Aut } \Gamma^{\prime}}\right)$ has connectivity one. Now $G$ is a primitive subgroup of Aut $\Gamma$, and thus Aut $\Gamma^{\prime}$, so the graph $\left(\Omega,\{\alpha, \beta\}^{G}\right)$ is a connected subgraph of $\left(\Omega,\{\alpha, \beta\}^{\text {Aut } \Gamma^{\prime}}\right)$, and therefore has connectivity one. Hence, the digraph $\Gamma:=\left(\Omega,(\alpha, \beta)^{G}\right)$ also has connectivity one. 
Lemma 3.5. ([6, Lemma 2.1.]) If $G$ acts primitively on the vertices, and transitively on the arcs, of a connectivity-one digraph $\Gamma$, and $\Lambda$ is any lobe of $\Gamma$ with at least three vertices, then the subgroup of Aut $\Lambda$ induced by the setwise stabiliser $G_{\{\Lambda\}}$ acts primitively on the vertices of $\Lambda$.

The following three lemmas are generalisations of several observations made in [4]. Although Möller restricts his attention to automorphism groups of graphs, for our purposes his arguments require only trivial modification.

Lemma 3.6. If $\Gamma$ is a locally finite connectivity-one orbital digraph of $G$ and $\alpha, \beta \in V \Gamma$ lie in the same lobe $\Lambda$ of $\Gamma$, then a lobe of $\Lambda^{\prime}:=$ $\left(V \Lambda,(\alpha, \beta)^{G_{\{\Lambda\}}}\right)$ is a lobe of $\Gamma^{\prime}:=\left(V \Gamma,(\alpha, \beta)^{G}\right)$.

Let $\Gamma$ be a locally finite orbital digraph of $G$ with more than one end. Fix $\alpha, \beta \in V \Gamma$ such that $\Gamma^{(1)}:=\left(V \Gamma,(\alpha, \beta)^{G}\right)$ has connectivity one. Let $\Lambda_{1}$ be a lobe of $\Gamma^{(1)}$ containing $\alpha$, and let $G^{(1)}:=G_{\left\{\Lambda_{1}\right\}}$. This group acts primitively on $V \Lambda_{1}$ by Lemma 3.5. If $\Lambda_{1}$ has more than one end then find $\beta_{2} \in \Lambda_{1}$ such that $\Gamma^{(2)}:=\left(V \Lambda_{1},\left(\alpha, \beta_{2}\right)^{G^{(1)}}\right)$ has connectivity one. Let $\Lambda_{2}$ be a lobe of $\Gamma^{(2)}$ containing $\alpha$ and put $G^{(2)}:=G_{\left\{\Lambda_{2}\right\}}$. Again we note this group acts primitively on $V \Lambda_{2}$ by Lemma 3.5. For $i \geq 2$, if $\Lambda_{i}$ has more than one end, find $\beta_{i+1} \in \Lambda_{i}$ such that $\Gamma^{(i+1)}:=\left(V \Lambda_{i},\left(\alpha, \beta_{i+1}\right)^{G^{(i)}}\right)$ has connectivity one. Let $\Lambda_{i+1}$ be a lobe of $\Gamma^{(i+1)}$ containing $\alpha$ and put $G^{(i+1)}:=G_{\left\{\Lambda_{i+1}\right\}}$. By Lemma 3.5. this group acts primitively on $V \Lambda_{i+1}$.

Lemma 3.7. There is an $n \geq 1$ such that $\Lambda_{n}$ has at most one end.

Lemma 3.8. For all integers $i \geq 1$, the digraph $\Lambda_{i}$ is a lobe of the orbital digraph $\left(V \Gamma,\left(\alpha, \beta_{i}\right)^{G}\right)$.

Proof of Theorem 3.3. Suppose $G$ has more than one permutation-end and no infinite subdegree.

If $\Gamma$ is a non-diagonal orbital digraph of $G$, then $\Gamma$ has more than one end. Furthermore, if $(\alpha, \beta) \in A \Gamma$, and $(\gamma, \alpha) \in A \Gamma$, then $\alpha$ is adjacent to at most $\left|\beta^{G_{\alpha}}\right|+\left|\gamma^{G_{\alpha}}\right|$ vertices in $\Gamma$. Since $G$ has no infinite subdegrees, this sum is finite, and since $G$ acts transitively on $V \Gamma$, this digraph, and indeed all orbital digraphs of $G$, are locally finite.

Thus, by Lemma 3.4, $G$ has a locally finite connectivity-one orbital digraph $\Gamma$. Using the notation described above, if we set $\Gamma^{(1)}:=\Gamma$ then, by Lemma 3.7, there exists a positive integer $n$ such that $\Lambda_{n}$ has at most one end. Put $\Gamma^{\prime}:=\left(V \Gamma,\left(\alpha, \beta_{n}\right)^{G}\right)$. By Lemma 3.8, $\Lambda_{n}$ is a lobe of $\Gamma^{\prime}$, and since $\Lambda_{n} \neq \Gamma^{\prime}$, we see $\Gamma^{\prime}$ has connectivity one. Furthermore, $G$ acts arc-transitively on $\Gamma^{\prime}$, so every lobe of $\Gamma^{\prime}$ is isomorphic to $\Lambda_{n}$, and thus has at most one end.

The structure of primitive directed digraphs with connectivity one is well known. 
Theorem 3.9. ([6, Theorem 2.6.]) Let $G$ be a vertex-transitive group of automorphisms of a connectivity-one digraph $\Gamma$ whose lobes have at least three vertices. If $G$ acts primitively on $V \Gamma$ and $\Lambda$ is some lobe of $\Gamma$, then $G_{\{\Lambda\}}$ is primitive and not regular on $V \Lambda$.

Recall that a digraph $\Gamma$ is automorphism-regular if Aut $\Gamma$ acts regularly on $V \Gamma$.

Theorem 3.10. ([6, Theorem 3.3.]) If $\Gamma$ is a vertex-transitive digraph with connectivity one, then it is primitive if and only if the lobes of $\Gamma$ are primitive but not automorphism-regular, pairwise isomorphic and each has at least three vertices.

Combining Theorem 3.3 , Theorem 3.9 and Theorem 3.10 we obtain the following.

Theorem 3.11. If $G$ is a primitive group of permutations of an infinite set $\Omega$ with more than one permutation-end and no infinite subdegree, then $G$ has a locally finite orbital digraph $\Gamma$ with connectivity one, whose lobes are primitive but not automorphism-regular, are pairwise isomorphic, have at least three vertices and at most one end. Furthermore, if $\Lambda$ is a lobe of $\Gamma$, then $G_{\{\Lambda\}}$ acts primitively but not regularly on $V \Lambda$.

If $G$ is a primitive group of permutations of an infinite set $\Omega$ and $\Gamma$ is a locally finite orbital digraph with connectivity one whose lobes have at most one end, then we shall call $\Gamma$ a canonical orbital digraph of $G$. If $\Gamma$ is such a digraph, and $\Lambda$ is a lobe of $\Gamma$ containing precisely one end $\epsilon$, then $\epsilon$ will be called a lobe-end of $\Gamma$. If an end $\epsilon$ is not a lobe-end of $\Gamma$, it will be called a tree-end, as it corresponds to an end of the block-cut-vertex tree of $\Gamma$.

Observe that $G$ permutes the lobe-ends of $\Gamma$ transitively. Indeed, $G$ acts arc-transitively on $\Gamma$, and each arc belongs to a unique lobe of $\Gamma$, so $G$ permutes the lobes, and therefore the lobe-ends, transitively.

Since $\Gamma$ has connectivity one, any thick ends of $\Gamma$ must be lobe-ends. Thus, if $\Gamma$ has a thick end then $G$ acts transitively on the thick ends of $\Gamma$.

\section{UNIQUENESS}

Recall that if $G$ is a primitive group and $\Gamma_{1}$ and $\Gamma_{2}$ are locally finite orbital digraphs of $G$ then there is a natural bijection between the ends of $\Gamma_{1}$ and the ends of $\Gamma_{2}$.

A geodesic between two connected vertices of a graph or digraph $\Gamma$ is a path of minimal length between them. If $T$ is a tree, and $\alpha, \beta \in V T$, there is exactly one geodesic in $T$ between $\alpha$ and $\beta$, which we denote by $[\alpha, \beta]_{T}$. If $\epsilon$ is an end of $T$, then there is precisely one half-line in $T$ that lies in $\epsilon$ with initial vertex $\alpha$. We denote this half-line by $[\alpha, \epsilon)_{T}$. A connected subgraph $C$ of $\Gamma$ is said to contain the end $\epsilon$ if, for every half-line $\left(\alpha_{i}\right)_{i \in \mathbb{N}}$ lying in $\epsilon$, we have $\alpha_{i} \in V C$ for all sufficiently large $i$. 

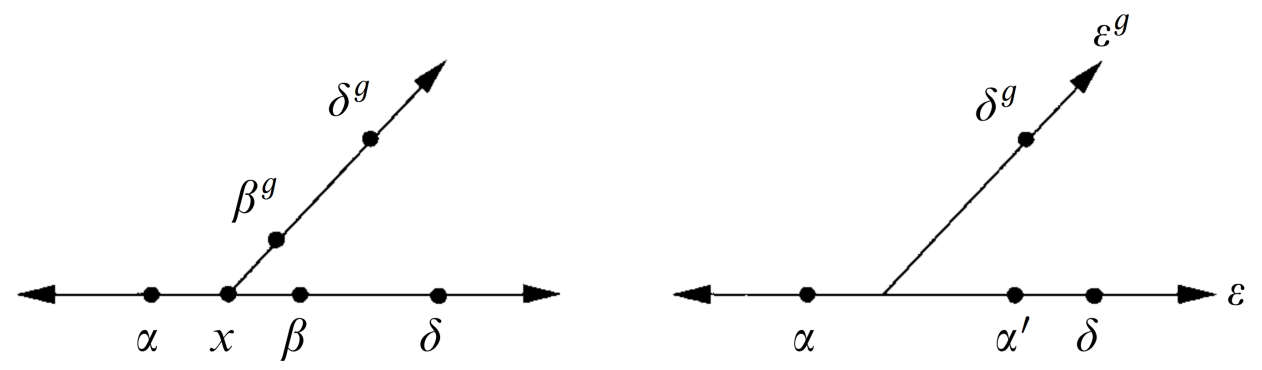

FiguRE 1 . The trees $T_{1}$ and $T_{2}$

Lemma 4.1. Suppose $\Gamma_{1}$ and $\Gamma_{2}$ are canonical orbital digraphs of a primitive group $G$. If $\epsilon$ is a lobe-end of $\Gamma_{1}$ then $\epsilon$ is a lobe-end of $\Gamma_{2}$.

Proof. Let $T_{1}$ and $T_{2}$ be the lobe-cut-vertex trees of $\Gamma_{1}$ and $\Gamma_{2}$ respectively. Suppose, for a contradiction, that there is a lobe-end $\epsilon$ of $\Gamma_{1}$ that is a tree-end of $\Gamma_{2}$. Since $\epsilon$ is a tree-end of $\Gamma_{2}$, it is an end of $T_{2}$. As it is a lobe-end of $\Gamma_{1}$, there exists a lobe $\Lambda_{1}$ of $\Gamma_{1}$ whose end is $\epsilon$, and a vertex $x \in V T_{1} \backslash V \Gamma_{1}$ corresponding to the lobe $\Lambda_{1}$.

The following argument is illustrated in Figure 1. Fix $\alpha \in V \Lambda_{1}$, and let $C$ be the connected component of $T_{2} \backslash\{\alpha\}$ containing $\epsilon$. Since $\epsilon$ is an end of $\Lambda_{1}$ and $\epsilon$ is contained in $C$, there must be infinitely many vertices in $V \Lambda_{1}$ that lie in $C$. Choose $\alpha^{\prime} \in V \Lambda_{1} \backslash\{\alpha\}$ such that $\alpha^{\prime} \in C$, and fix any vertex $\delta \in[\alpha, \epsilon)_{T_{2}} \cap\left[\alpha^{\prime}, \epsilon\right)_{T_{2}}$. Let $\beta$ be the vertex adjacent to $x$ in $[x, \delta]_{T_{1}}$. Since $\beta$ is adjacent to $x$ in $T_{1}$, we have $\beta \in V \Lambda_{1}$. If $\beta=\alpha$ then redefine $\alpha:=\alpha^{\prime}$. In this way, we can be sure $\beta \neq \alpha$.

Since $\beta \in V \Lambda_{1}$ and $G_{\left\{\Lambda_{1}\right\}}$ is primitive but not regular on $V \Lambda_{1}$, there is an element $g \in G_{\alpha,\left\{\Lambda_{1}\right\}}$ such that $\beta^{g} \neq \beta$. Now $\beta \in[\alpha, \delta]_{T_{1}}$, so $[\alpha, \delta]_{T_{1}} \neq[\alpha, \delta]_{T_{1}}^{g}$. However, $g \in G_{\alpha}$, so $[\alpha, \delta]_{T_{1}}^{g}=\left[\alpha, \delta^{g}\right]_{T_{1}}$ and therefore $\delta \neq \delta^{g}$. There is only one half-line in $T_{1}$ with initial vertex $\alpha$ contained in the end $\epsilon$, so if $g \in G_{\alpha, \epsilon}$ then $g$ must fix the half-line $[\alpha, \epsilon)_{T_{1}}$ pointwise. The vertex $\delta$ lies on this half-line, so $G_{\alpha, \epsilon} \leq G_{\delta}$. Since $\delta \neq \delta^{g}$, the automorphism $g$ thus cannot lie in $G_{\epsilon}$. This, however, is absurd, as $\epsilon$ is the end of $\Lambda_{1}$, and $g \in G_{\alpha,\left\{\Lambda_{1}\right\}} \leq G_{\alpha, \epsilon}$.

If $\Gamma$ is a locally finite connected graph or digraph, an automorphism $g \in$ Aut $\Gamma$ is called hyperbolic if $g$ fixes precisely two thin ends of $\Gamma$ and leaves no non-empty finite subset of $V \Gamma$ invariant.

Theorem 4.2. ([7, Proposition 3.4]) If $T$ is an undirected tree and $G$ is a group of automorphism of $T$ containing no hyperbolic elements, then either $G$ fixes some vertex or leaves some edge invariant, or $G$ fixes some end of the tree $T$.

Lemma 4.3. Suppose $G$ is a primitive group of permutations of an infinite set and $\Gamma_{1}$ and $\Gamma_{2}$ are canonical orbital digraphs of $G$. Let $T_{2}$ be the block-cut-vertex tree of $\Gamma_{2}$. If $\Lambda_{1}$ is a lobe of $\Gamma_{1}$ then there exists 
$x \in V T_{2}$ such that $G_{\left\{\Lambda_{1}\right\}} \leq G_{x}$, and for all $\alpha_{1}, \alpha_{2} \in V \Lambda_{1}$ we have $d_{T_{2}}\left(\alpha_{1}, x\right)=d_{T_{2}}\left(\alpha_{2}, x\right)$.

Proof. If $\Lambda_{1}$ is infinite, then it contains exactly one lobe-end $\epsilon$ of $\Gamma_{1}$, which by Lemma 4.1 is a lobe-end of $\Gamma_{2}$. Let $\Lambda_{2}$ be the lobe of $\Gamma_{2}$ whose end is $\epsilon$ and let $x$ be the vertex of $T_{2}$ corresponding to the lobe $\Lambda_{2}$. Then $G_{\left\{\Lambda_{1}\right\}}=G_{\epsilon}=G_{\left\{\Lambda_{2}\right\}}=G_{x}$, so we have $G_{\left\{\Lambda_{1}\right\}}$ fixes some vertex in $T_{2}$.

Now assume $V \Lambda_{1}$ is finite. Since $G_{\left\{\Lambda_{1}\right\}}$ cannot contain a hyperbolic element, we may use Theorem 4.2 to deduce that $G_{\left\{\Lambda_{1}\right\}}$ must fix a vertex of $T_{2}$, or leave some edge of $T_{2}$ invariant, or fix an end of $T_{2}$. In the latter two cases, the group must still fix some vertex of $T_{2}$. Indeed, since no element of $G$ may interchange two adjacent vertices of $T_{2}$, if the group $G_{\left\{\Lambda_{1}\right\}}$ leaves some edge $\{\alpha, x\}$ of $T_{2}$ invariant, then it must fix both $\alpha$ and $x$ pointwise. Furthermore, if $\epsilon$ is some end of $T_{2}$ fixed by the group $G_{\left\{\Lambda_{1}\right\}}$, take $x$ to be any vertex in $T_{2}$ such that the connected component of $T_{2} \backslash\{x\}$ containing the end $\epsilon$ contains no element in $V \Lambda_{1}$. Since $V \Lambda_{1}$ is finite, there are infinitely many choices for such a vertex. Then any half-line in $\epsilon$ whose root lies in $V \Lambda_{1}$ must contain the vertex $x$. Thus, if $G_{\left\{\Lambda_{1}\right\}}$ fixes the end $\epsilon$, it must also fix the vertex $x$.

Finally we observe that, since $G_{\left\{\Lambda_{1}\right\}}$ is transitive on $V \Lambda_{1}$, if $\alpha_{1}, \alpha_{2} \in$ $V \Lambda_{1}$ then $d_{T_{2}}\left(\alpha_{1}, x\right)=d_{T_{2}}\left(\alpha_{2}, x\right)$.

Henceforth, if $\Lambda_{1}$ is a lobe of $\Gamma_{1}$, the vertex $x$ in the above lemma will be referred to as the centroid of $\Lambda_{1}$ in $T_{2}$. Since the above arguments are symmetric in $\Gamma_{1}$ and $\Gamma_{2}$, we can also make reference to the centroid of $\Lambda_{2}$ in $T_{1}$, where $\Lambda_{2}$ is any lobe of $\Gamma_{2}$, and $T_{1}$ is the block-cut-vertex tree of $\Gamma_{1}$.

Let $\Omega$ be some fixed infinite set and let $G$ be a primitive group of permutations of $\Omega$. Given any locally finite primitive connectivity-one orbital digraph $\Gamma_{1}$ of $G$, if each vertex lies in $m$ distinct lobes and $\Lambda$ is a lobe of $\Gamma_{1}$, we write the digraph $\Gamma_{1}$ as $\Gamma(m, \Lambda)$. By Theorem 3.11 , all locally finite primitive connectivity-one orbital digraphs of $G$ can be written in this way. Since $G$ acts transitively on the set of lobes of $\Gamma(m, \Lambda)$, there is a natural equivalence relation on the set of such digraphs: the orbital digraph $\Gamma_{2}$ is equivalent to $\Gamma_{1}$ if $\Gamma_{2}=\Gamma\left(m, \Lambda^{\prime}\right)$ for some lobe $\Lambda^{\prime}$ of $\Gamma_{2}$ satisfying $V \Lambda=V \Lambda^{\prime}$. It is under this equivalence relation that a canonical orbital digraph of a primitive permutation group can be considered essentially unique.

Theorem 4.4. If $G$ is a primitive group of permutations of an infinite set $\Omega$ possessing no infinite subdegree, then the canonical orbital digraphs of $G$ are equivalent.

Proof. The group $G$ has at least one canonical orbital digraph by Theorem 3.11. Suppose $G$ has two canonical orbital digraphs $\Gamma_{1}=\Gamma\left(m_{1}, \Lambda_{1}\right)$ and $\Gamma_{2}=\Gamma\left(m_{2}, \Lambda_{2}\right)$. By Theorem 3.10, both $m_{1}$ and $m_{2}$ are at least 
two, and the digraphs $\Lambda_{1}$ and $\Lambda_{2}$ are primitive but not automorphismregular, and have at least three vertices. Let $T_{1}$ and $T_{2}$ be the block-cutvertex trees of $\Gamma_{1}$ and $\Gamma_{2}$ respectively. We wish to show there is a lobe $\Lambda_{2}^{\prime}$ of $\Gamma_{2}$ such that $V \Lambda_{1}=V \Lambda_{2}^{\prime}$. From this we will deduce that the digraphs $\Gamma_{1}$ and $\Gamma_{2}$ are equivalent. Set $d:=\sup \left\{d_{T_{2}}(\alpha, \beta) \mid \alpha, \beta \in V \Lambda_{1}\right\}$. This is finite, by Lemma 4.3 , so we may fix $\alpha, \beta \in V \Lambda_{1}$ such that $d_{T_{2}}(\alpha, \beta)=d$. Let $x_{2}$ be the vertex of $T_{2}$ that is adjacent to $\alpha$ in the line $[\alpha, \beta]_{T_{2}}$. Since $x_{2}$ is adjacent to $\alpha$ in $T_{2}$, it must lie in $V T_{2} \backslash V \Gamma_{2}$, and therefore corresponds to some lobe $\Lambda_{2}^{\prime}$ of $\Gamma_{2}$. Let $c_{1}$ be the centroid of $\Lambda_{1}$ in $T_{2}$.

We begin by showing $G_{\alpha,\left\{\Lambda_{1}\right\}}=G_{\alpha,\left\{\Lambda_{2}^{\prime}\right\}}$. Now $c_{1}$ and $\beta$ must lie in the same connected component of $T_{2} \backslash\{\alpha\}$, and $\beta$ and $x_{2}$ lie in the same component of $T_{2} \backslash\{\alpha\}$, so $c_{1}$ and $x_{2}$ are in the same component of $T_{2} \backslash\{\alpha\}$. Since $x_{2}$ is adjacent to $\alpha$ in $[\alpha, \beta]_{T_{2}}$, it follows that $x_{2} \in$ $\left[\alpha, c_{1}\right]_{T_{2}}$, and therefore $G_{\alpha, c_{1}} \leq G_{x_{2}}$. Thus

$$
G_{\alpha,\left\{\Lambda_{1}\right\}} \leq G_{\alpha, c_{1}} \leq G_{\alpha, x_{2}}=G_{\alpha,\left\{\Lambda_{2}^{\prime}\right\}} .
$$

Now choose $\gamma \in \Lambda_{2}^{\prime} \backslash\{\alpha\}$ and repeat this argument, replacing the vertex $\beta$ with $\gamma$, the tree $T_{2}$ with $T_{1}$ and the lobe $\Lambda_{1}$ with $\Lambda_{2}^{\prime}$. Whence, there exists a lobe $\Lambda_{1}^{\prime}$ of $\Gamma_{1}$ containing $\alpha$ such that $G_{\alpha,\left\{\Lambda_{2}^{\prime}\right\}} \leq G_{\alpha,\left\{\Lambda_{1}^{\prime}\right\}}$, and therefore

$$
G_{\alpha,\left\{\Lambda_{1}\right\}} \leq G_{\alpha,\left\{\Lambda_{2}^{\prime}\right\}} \leq G_{\alpha,\left\{\Lambda_{1}^{\prime}\right\}}
$$

Since $G_{\alpha}$ is transitive on the lobes of $\Gamma_{1}$ containing $\alpha$, there exists $g \in G_{\alpha}$ such that $\Lambda_{1}^{g}=\Lambda_{1}^{\prime}$. As there are only finitely many such lobes, $\Lambda_{1}^{g^{n}}=\Lambda_{1}$ for some natural number $n$. Now $G_{\alpha,\left\{\Lambda_{1}\right\}} \leq G_{\alpha,\left\{\Lambda_{1}\right\}^{g}}$, and therefore $G_{\alpha,\left\{\Lambda_{1}\right\}^{g}} \leq G_{\alpha,\left\{\Lambda_{1}\right\}^{g^{2}}}$. If we continue in this way we eventually obtain

$$
G_{\alpha,\left\{\Lambda_{1}\right\}} \leq G_{\alpha,\left\{\Lambda_{1}\right\}^{g}} \leq \cdots \leq G_{\alpha,\left\{\Lambda_{1}\right\}^{g^{n}}}
$$

however, $\Lambda_{1}^{g^{n}}=\Lambda_{1}$, so it follows that $G_{\alpha,\left\{\Lambda_{1}\right\}}=G_{\alpha,\left\{\Lambda_{1}\right\}^{g}}$. Thus

$$
G_{\alpha,\left\{\Lambda_{1}\right\}}=G_{\alpha,\left\{\Lambda_{2}^{\prime}\right\}} .
$$

We now show $G_{\left\{\Lambda_{1}\right\}}=G_{\left\{\Lambda_{2}^{\prime}\right\}}$. Let $\gamma$ be the vertex in $\left[x_{2}, \beta\right]_{T_{2}}$ adjacent to $x_{2}$, and observe $\gamma \in V \Lambda_{2}^{\prime}$. Now $G_{\left\{\Lambda_{2}^{\prime}\right\}}$ does not act regularly on $V \Lambda_{2}^{\prime}$, so there is an element $g \in G_{\alpha,\left\{\Lambda_{2}^{\prime}\right\}}$ such that $\gamma^{g} \neq \gamma$. Therefore $x_{2}$ is the only vertex in $\left[x_{2}, \beta\right]_{T_{2}} \cap\left[x_{2}, \beta^{g}\right]_{T_{2}}$. Thus $d_{T_{2}}\left(\beta, \beta^{g}\right)=d_{T_{2}}\left(\beta, x_{2}\right)+$ $d_{T_{2}}\left(\beta^{g}, x_{2}\right)=(d-1)+(d-1)=2 d-2$. Now $g \in G_{\alpha,\left\{\Lambda_{2}^{\prime}\right\}}=G_{\alpha,\left\{\Lambda_{1}\right\}}$, and $\beta \in V \Lambda_{1}$, so $\beta^{g} \in V \Lambda_{1}$. Furthermore, $d=d_{T_{2}}(\alpha, \beta)$ must be even, so if $d>2$, then $d \geq 4$, and therefore $d_{T_{2}}\left(\beta, \beta^{g}\right)=2 d-2>d$. This is not possible, as $d$ was chosen to be maximal. It must therefore be the case that $d=2$.

Since no two vertices of $\Lambda_{1}$ are at distance greater than $d$ in $T_{2}$, all vertices of $\Lambda_{1}$ are adjacent to $x_{2}$ in $T_{2}$, and therefore lie in $\Lambda_{2}^{\prime}$. Now $\Lambda_{1}$ contains at least three vertices, each of which is adjacent to $x_{2}$ in $T_{2}$, and so, since $G_{\left\{\Lambda_{1}\right\}}$ is transitive on $V \Lambda_{1}$, it must be the case that $G_{\left\{\Lambda_{1}\right\}}$ fixes the vertex $x_{2}$, and therefore fixes $V \Lambda_{2}^{\prime}$ setwise. Thus $G_{\left\{\Lambda_{1}\right\}} \leq G_{\left\{\Lambda_{2}^{\prime}\right\}}$. If 
$G_{\left\{\Lambda_{1}\right\}} \neq G_{\left\{\Lambda_{2}^{\prime}\right\}}$ then $G_{\alpha,\left\{\Lambda_{2}^{\prime}\right\}}=G_{\alpha,\left\{\Lambda_{1}\right\}}<G_{\left\{\Lambda_{1}\right\}}<G_{\left\{\Lambda_{2}^{\prime}\right\}}$, which cannot happen since $G_{\left\{\Lambda_{2}^{\prime}\right\}}$ is primitive on $V \Lambda_{2}$. Hence $G_{\left\{\Lambda_{1}\right\}}=G_{\left\{\Lambda_{2}^{\prime}\right\}}$.

Finally, since $G_{\left\{\Lambda_{1}\right\}}$ and $G_{\left\{\Lambda_{2}^{\prime}\right\}}$ are transitive on $V \Lambda_{1}$ and $V \Lambda_{2}^{\prime}$ respectively, and $\alpha \in V \Lambda_{1} \cap V \Lambda_{2}^{\prime}$, we have $V \Lambda_{2}^{\prime}=\alpha^{G_{\left\{\Lambda_{2}^{\prime}\right\}}}=\alpha^{G_{\left\{\Lambda_{1}\right\}}}=V \Lambda_{1}$.

Now, $\Lambda_{1}$ is vertex-transitive, so the out-valency and in-valency of each vertex must be non-zero. Since $\Gamma_{1}$ is arc-transitive, this implies that $G_{\alpha}$ is transitive on the set of lobes of $\Gamma_{1}$ that contain $\alpha$. Similarly, $G_{\alpha}$ is transitive on the set of lobes of $\Gamma_{2}$ containing $\alpha$. Whence, $m_{1}=$ $\left|G_{\alpha}: G_{\alpha,\left\{\Lambda_{1}\right\}}\right|=\left|G_{\alpha}: G_{\alpha,\left\{\Lambda_{2}^{\prime}\right\}}\right|=m_{2}$. Therefore $\Gamma_{2}=\Gamma\left(m_{1}, \Lambda_{2}^{\prime}\right)$ and $V \Lambda_{2}^{\prime}=V \Lambda_{1}$, so the digraphs $\Gamma_{1}$ and $\Gamma_{2}$ are equivalent.

It is now possible to describe every locally finite connectivity-one orbital digraph of a primitive group $G$.

Theorem 4.5. If $G$ is a primitive group of permutations of an infinite set $\Omega$ with more than one permutation-end and no infinite subdegree, then $G$ has a canonical orbital digraph $\Gamma(m, \Lambda)$, and the canonical orbital digraphs of $G$ are precisely the digraphs $\Gamma\left(m, \Lambda^{\prime}\right)$, where $\Lambda^{\prime}$ is an orbital digraph of $G_{\{\Lambda\}}$ acting on the set $V \Lambda$.

Proof. Suppose $G$ has a locally finite orbital digraph with more than one end. By Theorem 3.11, $G$ has a canonical orbital digraph $\Gamma(m, \Lambda)$. Set $\Gamma:=\Gamma(m, \Lambda)$, and fix vertices $\alpha, \beta \in V \Lambda$ such that $\Gamma=\left(\Omega,(\alpha, \beta)^{G}\right)$. Suppose $\Gamma^{\prime}$ is also a canonical orbital digraph of $G$. We show there exists an orbital digraph $\Lambda^{\prime}$ of $G_{\{\Lambda\}}$ acting on $V \Lambda$ such that $\Lambda^{\prime}$ is a lobe of $\Gamma^{\prime}$ and $\Gamma^{\prime}=\Gamma\left(m, \Lambda^{\prime}\right)$. By Theorem 4.4, the digraph $\Gamma^{\prime}$ must be equivalent to $\Gamma$, so there exists a lobe $\Lambda^{\prime}$ of $\Gamma^{\prime}$ such that $\Gamma^{\prime}=\Gamma\left(m, \Lambda^{\prime}\right)$ and $V \Lambda^{\prime}=V \Lambda$. Let $\left(\alpha, \beta^{\prime}\right)$ be an arc in $\Lambda^{\prime}$. Since $\Gamma^{\prime}$ is arc-transitive, $\Gamma^{\prime}=\left(\Omega,\left(\alpha, \beta^{\prime}\right)^{G}\right)$. If $(\gamma, \delta)$ is any arc in $\Lambda^{\prime}$, then there exists an element $g \in G$ such that $(\gamma, \delta)=\left(\alpha, \beta^{\prime}\right)^{g}$, but such an automorphism must fix the lobe $\Lambda^{\prime}$, and therefore lies in $G_{\left\{\Lambda^{\prime}\right\}}=G_{\{\Lambda\}}$. Hence $\Lambda^{\prime}$ is an orbital digraph of $G_{\{\Lambda\}}$ acting on the set $V \Lambda$.

Conversely, suppose $\Lambda^{\prime}$ is an orbital digraph of $G_{\{\Lambda\}}$ acting on $V \Lambda$. We show the connectivity-one digraph $\Gamma\left(m, \Lambda^{\prime}\right)$ is a canonical orbital digraph of $G$.

It is simple to check that $G \leq$ Aut $\Gamma\left(m, \Lambda^{\prime}\right)$, so we begin by showing $G$ is arc-transitive on $\Gamma\left(m, \Lambda^{\prime}\right)$. Suppose $\Lambda_{1}^{\prime}$ and $\Lambda_{2}^{\prime}$ are lobes of $\Gamma\left(m, \Lambda^{\prime}\right)$. Then there exist lobes $\Lambda_{1}$ and $\Lambda_{2}$ of $\Gamma(m, \Lambda)$ such that $V \Lambda_{1}=V \Lambda_{1}^{\prime}$ and $V \Lambda_{2}=V \Lambda_{2}^{\prime}$. Since $G$ acts arc-transitively on $\Gamma(m, \Lambda)$, it acts transitively on its lobes. Therefore, there exists an automorphism $g \in G$ such that $\Lambda_{1}^{g}=\Lambda_{2}^{g}$. Since $G \leq \operatorname{Aut} \Gamma\left(m, \Lambda^{\prime}\right)$, we must also have $\Lambda_{1}^{\prime g}=\Lambda_{2}^{\prime}$, so $G$ permutes the lobes of $\Gamma\left(m, \Lambda^{\prime}\right)$ transitively. Thus $G$ is transitive on the lobes of $\Gamma(m, \Lambda)$ and acts arc-transitively on each lobe, so it must act arc-transitively on the whole digraph $\Gamma\left(m, \Lambda^{\prime}\right)$.

It remains to show the lobes of $\Gamma\left(m, \Lambda^{\prime}\right)$ have at most one end. This follows immediately from Lemma 2.2 , since both $\Lambda$ and $\Lambda^{\prime}$ are orbital digraphs of $G_{\{\Lambda\}}$ acting on $V \Lambda$, they must have the same ends. 
Hence, $\Gamma(m, \Lambda)$ is a connectivity-one orbital digraph of $G$ in which each lobe has at most one end, and is therefore a canonical orbital digraph of the group $G$.

Thus, for any primitive group $G$ possessing a canonical orbital digraph $\Gamma$, when speaking of the permutation-ends of $G$ as being the ends of $\Gamma$, it makes sense to refer to the lobe-ends and tree-ends of $G$ as being, respectively, the lobe-ends and tree-ends of its canonical orbital digraph. This can be extended to include any group $G$ with just one permutation-end $\epsilon$, by defining the lobe-end of $G$ to be the end $\epsilon$.

That a whole class of infinite primitive permutation groups possessing no infinite subdegree should each have an orbital digraph whose structure is tree-like is a property that will be explored and exploited in a future paper by the author. In particular, this result will be fundamental in the examination of the possible rates of subdegree growth of infinite primitive permutation groups.

The concluding section of this paper is conceived as a simple illustration of just one way in which the existence of the canonical orbital digraph may be used to easily obtain a deep understanding of the structure of infinite primitive groups possessing no infinite subdegree, through the application of Bass and Serre's theory on trees.

\section{The STRUCTURE OF INFINITE PRIMITIVE GROUPS}

A common example of an amalgamated free product is when there are two groups $G_{1}$ and $G_{2}$, with subgroups $H_{1}$ and $H_{2}$ that are isomorphic via $\varphi: H_{1} \rightarrow H_{2}$. In this case the free product of $G_{1}$ and $G_{2}$ with amalgamated subgroup $H_{1}$ is denoted by $G_{1} *_{H_{1}} G_{2}$.

Let $G$ be a group acting on a digraph $\Gamma$. An inversion is a pair consisting of an element $g \in G$ and an $\operatorname{arc}(x, y) \in A \Gamma$ such that $(x, y)^{g}=(y, x)$; if there is no such pair the group $G$ is said to act without inversion on $\Gamma$. If $G$ acts on $\Gamma$ without inversion, we define the quotient digraph $G / \Gamma$ to be the digraph whose vertex and arc set are the quotients of $V \Gamma$ and $A \Gamma$ respectively under the action of $G$; a fundamental domain of $\Gamma \bmod G$ is a subgraph of $\Gamma$ that is isomorphic to $G / \Gamma$. A segment of $\Gamma$ is a subgraph of $\Gamma$ consisting two adjacent vertices and an arc of $\Gamma$ between them.

Theorem 5.1. ([5, Theorem 6]) Let $G$ be a group acting on a digraph $\Gamma$ and let $P=\{\{x, y\},(x, y)\}$ be a segment of $\Gamma$. If $P$ is a fundamental domain of $\Gamma \bmod G$ then $\Gamma$ is a tree if and only if $G \cong G_{x} *_{G_{x, y}} G_{y}$.

Using this powerful theorem, we may determine the structure of infinite primitive permutation groups with more than one permutationend and no infinite subdegree. 
Theorem 5.2. If $G$ is an infinite primitive group of permutations of an infinite set $\Omega$ with more than one permutation-end and no infinite subdegree, then $G$ has a canonical orbital digraph $\Gamma(m, \Lambda)$ and

$$
G \cong G_{\alpha} *_{G_{\alpha,\{\Lambda\}}} G_{\{\Lambda\}},
$$

where $\alpha \in V \Lambda$, and $G_{\alpha,\{\Lambda\}}$ is a non-trivial maximal proper subgroup of $G_{\{\Lambda\}}$ that fixes no element in $V \Lambda \backslash\{\alpha\}$.

Proof. Let $G$ be an infinite primitive group possessing a finite suborbit whose pair is also finite, and suppose $G$ has an orbital digraph with more than one end. By Theorem 3.11, $G$ has a canonical orbital digraph $\Gamma$ of the form $\Gamma(m, \Lambda)$, where $m \geq 2$ and $\Lambda$ is a primitive but not automorphism-regular digraph, with at least three vertices and at most one end. Let $T$ be the block-cut-vertex tree of $\Gamma$. Then $G \leq$ Aut $T$. There is a natural bipartition of $T$, one part containing the vertices of $\Gamma$, and the other containing vertices corresponding to the lobes of $\Gamma$. Since $G$ preserves this bipartition, the group $G$ acts on $T$ without inversion, and has two orbits on the vertices of $T$.

Let $x \in V T$ correspond with the lobe $\Lambda$ of $\Gamma$, and fix $\alpha \in V \Lambda$. Then $(\alpha, x)$ is an $\operatorname{arc}$ in $T$. If we define $T^{\prime}:=\left(V T,(\alpha, x)^{G}\right)$, then $T^{\prime}$ is an arctransitive tree upon which $G$ acts without inversion, with fundamental domain the segment $\{\{\alpha, x\},(\alpha, x)\}$. Hence, by Theorem 5.1.

$$
G \cong G_{\alpha} *_{G_{\alpha, x}} G_{x} .
$$

Since $\Lambda$ is the lobe of $\Gamma$ corresponding to the vertex $x$ of $T$, the stabiliser $G_{x}$ is equal to $G_{\{\Lambda\}}$. As $G_{\{\Lambda\}}$ acts primitively on $V \Lambda$, the group $G_{\alpha,\{\Lambda\}}$ is maximal in $G_{\{\Lambda\}}$. By Theorem 3.9. $G_{\alpha,\{\Lambda\}}$ fixes no vertex in $V \Lambda \backslash$ $\{\alpha\}$.

This paper forms part of the author's DPhil thesis, completed at the University of Oxford, under the supervision of Peter Neumann. The author would like to thank Dr Neumann for his help and guidance. The author would also like to thank the EPSRC for generously funding this research.

\section{REFERENCES}

[1] W. Dicks and M. J. Dunwoody, Groups acting on graphs, Cambridge Studies in Advanced Mathematics 17 (Cambridge University Press, Cambridge, 1989).

[2] R. G. Möller, 'Ends of graphs II', Math. Proc. of the Cambridge Philos. Soc. (3) 111 (1992) 455-460.

[3] R. G. Möller, 'Groups acting on locally finite graphs - a survey of the infinitely ended case', Groups '93, Galway / St. Andrews, Vol. 2, London Mathematical Society Lecture Note Series (Cambridge Univ. Press, Cambridge, 1993), pp. 426-456.

[4] R. G. Möller, 'Primitivity and ends of graphs', Combinatorica (4) 14 (1994) 477-484.

[5] J-P. Serre, Trees (Springer-Verlag, Berlin, 2003). 
[6] S. M. Smith, 'Infinite primitive directed graphs', to appear, http://arxiv.org/abs/math.CO/0602011.

[7] J. Tits, 'Sur le groupe des automorphismes d'un arbre', Essays on topology and related topics, Mémoires dédiés à Georges de Rham (eds A. Haefliger and R. Narasimhan, Springer-Verlag, New York, 1970), pp. 188-211.

[8] M. E. Watkins, 'Ends and automorphisms of infinite graphs', Graph symmetry (eds G. Hahn and G. Sabidussi), NATO Adv. Sci. Inst. Ser. C Math. Phys. Sci. 497 (Kluwer Acad. Publ., Dordrecht, 1997), pp. 379-414. 